\title{
Absence of residual tumor tissue after Gamma Knife radiosurgery followed by resection of a vestibular schwannoma: illustrative case
}

\author{
Assaf Berger, MD, ${ }^{1}$ Kristyn Galbraith, MD, ${ }^{2}$ Matija Snuderl, MD, ${ }^{2}$ John G. Golfinos, MD, ${ }^{1}$ and Douglas Kondziolka, MD ${ }^{1}$ \\ Departments of ${ }^{1}$ Neurosurgery and ${ }^{2}$ Pathology, NYU Langone Medical Center, New York University, New York, New York
}

BACKGROUND Late pathology after vestibular schwannoma radiosurgery is uncommon. The authors presented a case of a resected hemorrhagic mass 13 years after radiosurgery, when no residual tumor was found.

OBSERVATIONS A 56-year-old man with multiple comorbidities, including myelodysplastic syndrome cirrhosis, received Gamma Knife surgery for a left vestibular schwannoma. After 11 years of stable imaging assessments, the lesion showed gradual growth until a syncopal event occurred 2 years later, accompanied by progressive facial weakness and evidence of intralesional hemorrhage, which led to resection. However, histopathological analysis of the resected specimen showed hemorrhage and reactive tissue but no definitive residual tumor.

LESSONS This case demonstrated histopathological evidence for the role of radiosurgery in complete elimination of tumor tissue. Radiosurgery for vestibular schwannoma carries a rare risk for intralesional hemorrhage in select patients.

https://thejns.org/doi/abs/10.3171/CASE21614

KEYWORDS vestibular schwannoma; radiosurgery; hemorrhage; case report

Stereotactic radiosurgery leads to tumor growth arrest and gradual reduction in tumor volume for most patients who undergo the procedure for a vestibular schwannoma. Postradiosurgery resection is uncommon and is usually performed for clear tumor recurrence. Pathology almost invariably shows recurrent tumor with reactive changes, including mixed chronic inflammatory effects and abnormal vasculature with focal proliferation. ${ }^{1-3}$ Although tumors can show significant tumor regression on high-quality imaging, complete regression of a tumor is rare after radiosurgery. Resection is usually not required; therefore, histological or autopsy analyses of post-Gamma Knife surgery tissues are rarely available. ${ }^{2}$ Intratumoral hemorrhage is rare and can occur as part of the response to radiosurgery, more frequently in patients with a coagulation disorder. In this report, we provide details regarding a patient after radiosurgery who developed an intralesional hemorrhage. The hemorrhage was resected without evidence of a residual tumor, suggesting that expansion was solely due to the hemorrhage and reactive tissue.

\section{Illustrative Case}

The patient was a 56-year-old man who was diagnosed with a left vestibular schwannoma that presented with left-sided tinnitus and hearing loss (Fig. 1). He had multiple comorbidities, including hypertension, diabetes mellitus, myelodysplastic syndrome, and a liver transplant for cirrhosis that required immunosuppression. The patient was not a suitable candidate for resection, and in 2007, the lesion was treated with 13 Gy Gamma Knife surgery. Serial imaging displayed tumor control for 11 years until a scan showed a slight asymptomatic interval increase.

Meanwhile, the patient developed non-Hodgkin lymphoma, which was treated with rituximab, accompanied by anemia, thrombocytopenia, and leukopenia that required treatments with colony-stimulating factors and erythropoietin alpha. He also developed paraparesis with a plausible diagnosis of transverse myelitis, which required him to use a wheelchair. Anticoagulation for deep vein thrombosis (DVT) prophylaxis was initiated. 


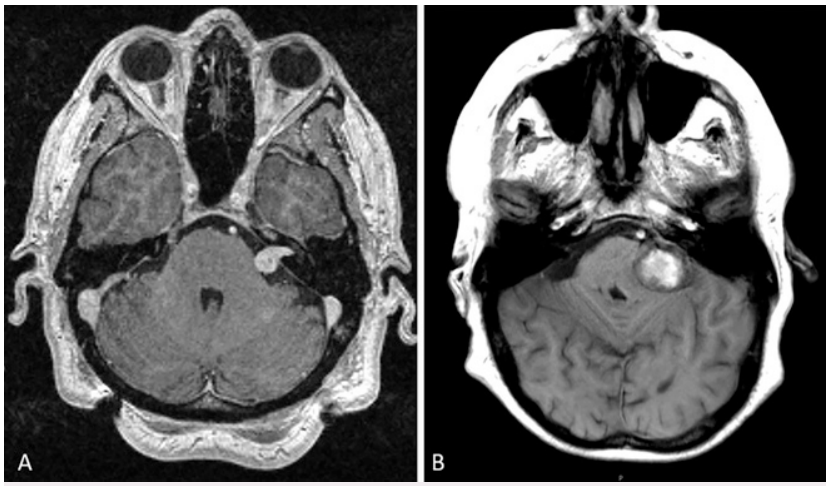

FIG. 1. Magnetic resonance imaging of the patient's left vestibular schwannoma on the day of radiosurgery (A, with contrast) and 13 years later, on the day of hemorrhagic mass expansion $(B$, without contrast).

Thirteen years after radiosurgery, he presented after a syncopal episode and persistent left ear pain. Computed tomography of his head demonstrated slight mass effect on brachium pontis with atypical calcification. He also developed left-sided facial spasm, which progressed to weakness despite steroid treatment. Several days later, another interval increase was documented, this time with associated precontrast T1 enhancement, which was suggestive of hemorrhage. Despite decreasing anticoagulant therapy, further expansion of the hemorrhagic lesion was noted, together with worsening facial weakness. He received a House-Brackmann grade of IV, which was accompanied by a left-sided facial numbness in the V2 and V3 distributions (Fig. 1). Because of the clinical deterioration, a translabyrinthine approach was subsequently performed for surgical removal, with the addition of facial reanimation surgery.

The patient recovered well, and the pathological specimen revealed a mixture of acute and remote hemorrhage, reactive granulation tissue with abundant fibroblasts, and rare scattered S-100-positive cells but without definitive vestibular schwannoma (Fig. 2).

\section{Discussion}

Here we describe an unusual case of a vestibular schwannoma that was treated by radiosurgery and developed delayed intralesional hemorrhage, necessitating its resection. It provided a unique insight into the histopathological findings of postradiosurgery specimens as well as the evolution of hemorrhages in vestibular schwannoma lesions and their risk factors.

\section{Observations}

Although histopathology after radiosurgery is not frequently accessible, a few studies have described the findings of postradiosurgical specimens. Many promote theories of a dose- and timedependent pattern of changes in treated tissue, in which postradiosurgery tumor changes occur over months to years after treatment. $^{1,2,4-6}$ In the months after radiosurgery, coagulation necrosis, apoptosis, interstitial edema, and reactive astrogliosis occur. A variety of vascular changes and sometimes less predictable adverse events, including cysts and aneurysms, may occur. ${ }^{2,7,8}$ In the delayed phase, scar tissue begins to replace the coagulation necrosis. ${ }^{1,9,10}$ Notably, our patient's pathology showed marked fibroblastic scarring process, which was nevertheless disrupted by the acute hemorrhage, leading to fresh granulation tissue.

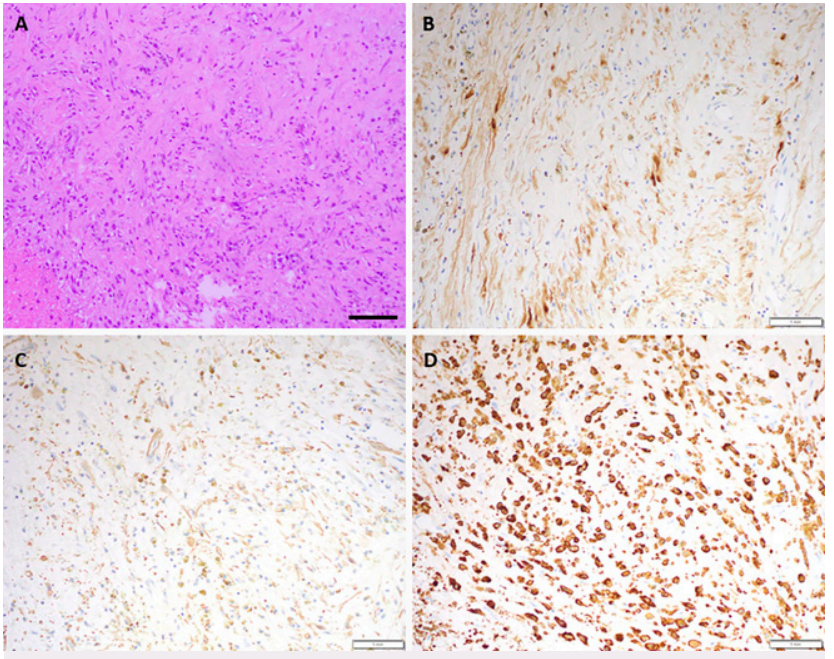

FIG. 2. Histology of granulation tissue. A: H\&E stain at $\times 20$ magnification of myofibroblasts with vascular proliferation and histiocytic inflammation consistent with granulation tissue. B-D: Immunohistochemical stains show that few residual cells express $S 100$ (B), myofibroblasts within the granulation tissue express SMA $(C)$, and extensive histiocytic inflammation is highlighted by CD168 (D).

Intratumoral hemorrhage can occur in both naïve and treated vestibular schwannomas, in which tumor growth control rates exceed $90 \%$ after radiosurgery. ${ }^{11}$ Various descriptions of vestibular schwannomas with intratumoral hemorrhage have been reported, and they typically present with acute symptom progression, $9,10,12-15$ including headache, disequilibrium, and rapidly progressive hearing loss. ${ }^{10,16}$ Although less common, facial and trigeminal nerve deficits can also occur. Management is based on the urgency of the situation. ${ }^{17}$ In our high-risk surgical patient who initially was stable, the primary choice was observation. As clinical and radiological deterioration occurred, we opted for surgical intervention.

The evolution of rare intralesional hemorrhage in vestibular schwannoma has been previously studied. Sughrue et al. found that many tumors contained microhemorrhages. ${ }^{5}$ Of the specimens studied, $23 \%$ were composed of blood in more than a quarter of the volume, and hemorrhage comprised more than half of the volume in $4 \%$ of tumors. The histopathological analysis by Liu et al. agreed with the high rate of intratumoral microhemorrhage, mentioning that hemosiderin deposition occurred in more than half of the 18 schwannomas analyzed. ${ }^{1}$ Although microhemorrhages in vestibular schwannoma are frequent, a larger hemorrhage and continued bleeding, as in the case of our patient, likely require a secondary factor. A two-hit phenomenon has been discussed in previous reports of vestibular schwannoma with intratumoral hemorrhage. Niknafs et al. summarized several extratumoral factors that could exacerbate microhemorrhages, including hypertension, pregnancy, head trauma, and antithrombotic agents. ${ }^{10,16}$ In our patient, underlying radiation changes could provide the initial hit, which was exacerbated by secondary factors, most notably numerous hematological comorbidities, including immunosuppression for his liver transplant, non-Hodgkin lymphoma, myelodysplastic syndrome and low platelets, and anticoagulation for DVT prophylaxis. According to the study by Carlson et al., there could be a predicted 25 -fold increased risk of intratumoral hemorrhage in persons with compromised coagulation. ${ }^{16}$ 


\section{Lessons}

Our case demonstrates histopathological evidence of a virtually complete regression of vestibular schwannoma after radiosurgery with reactive changes. However, radiosurgery for vestibular schwannoma carries a potential risk for intralesional hemorrhage, which may be more pronounced in patients with hematological comorbidities.

\section{Acknowledgments}

We wish to recognize the fine work of Dev Patel in contributing to this study.

\section{References}

1. Liu A, Wang JM, Li GL, et al. Clinical and pathological analysis of benign brain tumors resected after Gamma Knife surgery. J Neurosurg. 2014;121(suppl):179-187.

2. Hirato M, Hirato J, Zama A, et al. Radiobiological effects of gamma knife radiosurgery on brain tumors studied in autopsy and surgical specimens. Stereotact Funct Neurosurg. 1996;66(suppl 1):4-16.

3. Linskey ME, Martinez AJ, Kondziolka D, et al. The radiobiology of human acoustic schwannoma xenografts after stereotactic radiosurgery evaluated in the subrenal capsule of athymic mice. J Neurosurg. 1993;78(4):645-653.

4. Anniko M, Arndt J, Norén G. The human acoustic neurinoma in organ culture. II. Tissue changes after gamma irradiation. Acta Otolaryngol. 1981;91(3-4):223-235.

5. Sughrue ME, Kaur R, Kane AJ, et al. Intratumoral hemorrhage and fibrosis in vestibular schwannoma: a possible mechanism for hearing loss. J Neurosurg. 2011;114(2):386-393.

6. Kwon Y, Khang SK, Kim CJ, Lee DJ, Lee JK, Kwun BD. Radiologic and histopathologic changes after Gamma Knife radiosurgery for acoustic schwannoma. Stereotact Funct Neurosurg. 1999;72 (suppl 1):2-10.

7. Park KY, Ahn JY, Lee JW, Chang JH, Huh SK. De novo intracranial aneurysm formation after Gamma Knife radiosurgery for vestibular schwannoma. J Neurosurg. 2009;110(3):540-542.

8. Murakami K, Jokura H, Kawagishi J, Watanabe M, Tominaga T. Development of intratumoral cyst or extratumoral arachnoid cyst in intracranial schwannomas following gamma knife radiosurgery. Acta Neurochir (Wien). 2011;153(6):1201-1209.

9. Grady C, Tanweer O, Zagzag D, Jafar JJ, Huang PP, Kondziolka D. Delayed hemorrhage from the tissue of an occluded arteriovenous malformation after stereotactic radiosurgery: report of 3 cases. J Neurosurg. 2017;126(6):1899-1904.
10. Niknafs YS, Wang AC, Than KD, Etame AB, Thompson BG, Sullivan SE. Hemorrhagic vestibular schwannoma: review of the literature. World Neurosurg. 2014;82(5):751-756.

11. Chopra R, Kondziolka D, Niranjan A, Lunsford LD, Flickinger JC. Long-term follow-up of acoustic schwannoma radiosurgery with marginal tumor doses of 12 to $13 \mathrm{~Gy}$. Int J Radiat Oncol Biol Phys. 2007;68(3):845-851.

12. Thombre B, Sadashiva N, Krishnan JB, Prabhuraj AR, Rao KN, Arima A. Symptomatic post-radiosurgery intratumoral hemorrhage in a case of vestibular schwannoma: a case report and review of the literature. Stereotact Funct Neurosurg. 2019;97(5-6):399-403.

13. Suzuki H, Toyoda S, Muramatsu M, Shimizu T, Kojima T, Taki W. Spontaneous haemorrhage into metastatic brain tumours after stereotactic radiosurgery using a linear accelerator. J Neurol Neurosurg Psychiatry. 2003;74(7):908-912.

14. Karampelas I, Alberico RA, Plunkett RJ, Fenstermaker RA. Intratumoral hemorrhage after remote subtotal microsurgical resection and Gamma Knife radiosurgery for vestibular schwannoma. Acta Neurochir (Wien). 2007;149(3):313-317.

15. Dehdashti AR, Kiehl TR, Guha A. Vestibular schwannomas presenting with haemorrhage: clinical presentation and histopathological evaluation of an unusual entity. $\mathrm{Br} J$ Neurosurg. 2009;23(4):431-436.

16. Carlson ML, Tombers NM, Driscoll CLW, et al. Clinically significant intratumoral hemorrhage in patients with vestibular schwannoma. Laryngoscope. 2017;127(6):1420-1426.

17. Kondziolka D, Mousavi SH, Kano H, Flickinger JC, Lunsford LD. The newly diagnosed vestibular schwannoma: radiosurgery, resection, or observation? Neurosurg Focus. 2012;33(3):E8.

\section{Disclosures}

The authors report no conflict of interest concerning the materials or methods used in this study or the findings specified in this paper.

\section{Author Contributions}

Conception and design: Berger, Golfinos, Kondziolka. Acquisition of data: Galbraith, Snuderl, Golfinos, Kondziolka. Analysis and interpretation of data: all authors. Drafting the article: Berger, Snuderl, Kondziolka. Critically revising the article: Berger, Snuderl, Golfinos, Kondziolka. Reviewed submitted version of manuscript: all authors. Approved the final version of the manuscript on behalf of all authors: Berger. Administrative/technical/material support: Kondziolka.

\section{Correspondence}

Assaf Berger: NYU Langone Medical Center, New York University, New York, NY. assaf.berger@nyulangone.org. 